Revista de Derecho

\title{
Las guardianas del agua y su participación en la gestión comunitaria de los recursos hídricos. Un análisis de la normativa ecuatoriana
}

Women water guardians and their participation in communitarian management. An analysis of the Ecuadorian normative

\author{
Andrés Martínez-Moscoso \\ Director de Posgrados e Investigaciones \\ Facultad de Jurisprudencia de la Universidad de Cuenca, Ecuador \\ andres.martinez@ucuenca.edu.ec \\ ORCID: 0000-0002-8952-0680 \\ Adriana Abril Ortiz \\ Asistente de investigación \\ Departamento de Investigación en Espacio y Población \\ Universidad de Cuenca, Ecuador \\ adriana.abril@ucuenca.edu.ec \\ ORCID: 0000-0002-7405-8040
}

DOI: https://doi.org/10.32719/26312484.2020.34.4

Fecha de recepción: 30 de octubre de 2019

Fecha de aceptación: 15 de enero de 2020

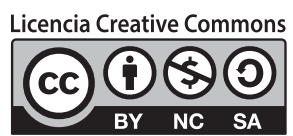




\section{RESUMEN}

El reconocimiento del agua como derecho humano y fundamental en la República del Ecuador trajo consigo un sinnúmero de retos, entre ellos la incorporación de un enfoque de género en la gestión comunitaria de los recursos hídricos. Esta situación ha sido recogida tanto en la ley como en los instrumentos de políticas públicas, pese a lo cual en la práctica aún la brecha de una real participación es elevada, debido a los roles de género que subsisten. El artículo realiza un estudio normativo de los instrumentos internacionales de protección de derechos humanos y la legislación ecuatoriana, respecto a las incorporaciones tanto del derecho humano al agua como de la gestión comunitaria, desde una perspectiva de género. Del mismo modo, incorpora los resultados de estudios de caso, en los que se comprueba que la participación libre, activa y significativa de la mujer en estos sistemas comunitarios, en el plano internacional y local, todavía no se logra en su totalidad. El trabajo concluye que la incorporación de las mujeres en la gestión y toma de decisiones de las juntas de agua de consumo humano no solo se hace a través de una participación que elimine las barreras físicas, sino también sociales, las cuales impiden que ellas puedan tener un rol protagónico al interior de las organizaciones, de manera activa cuanto pasiva.

PALABRAS Clave: Derechos Humanos; agua; participación de la mujer; gestión de los recursos hídricos; mujer rural; mujer y desarrollo.

\section{ABSTRACT}

The recognition of water as a human and fundamental right in Ecuador, implied countless challenges, including the incorporation of the gender approach in community management of water resources. This necessity has been acknowledged in the law, as well as in public policies, although in practice there is still a gap in the real participation of women due to persistent gender roles that exclude them from the decision making process. This paper develops a normative study, from international human rights instruments to Ecuadorian legislation regarding the incorporation of the human right to water, as well as the implementation of a gender perspective in water management. It also includes case studies, which show that active, free and meaningful participation of women in these communitarian systems is not yet fully achieved. After this analysis and the following discussion, the work concludes that the integration of women in management and decisionmaking of communitarian water committees, is not only done by eliminating physical, but also social barriers to participate, which could prevent women from having a leading role within their communities, in an active and passive form.

KEYWORDS: Human rights; water; women participation; water resources management; Rural women; women and development. 


\section{INTRODUCCIÓN}

T a incorporación del agua como un derecho humano y fundamental es de nueva data en el ámbito ecuatoriano. La Constitución de la República del Ecuador de 2008 reconoció el derecho al agua como parte de los derechos del Buen Vivir, a la par que, de manera transversal, incorporó la obligación del Estado de formular y ejecutar políticas para la igualdad entre mujeres y hombres mediante la incorporación del enfoque de género.

Por su parte, un hito importante en el reconocimiento del derecho humano al agua constituye la Observación General n. ${ }^{\circ} 15$ realizada por el Comité de Derechos Económicos, Sociales y Culturales, en la que se reconoció de manera expresa que "el derecho humano al agua es indispensable para vivir dignamente y es condición previa para la realización de otros derechos humanos". ${ }^{1}$ Sin embargo, fue hasta 2010 que el sistema de protección universal de los derechos humanos lo reconoció como prerrogativa fundamental para el pleno disfrute de la vida, en el marco de los derechos económicos sociales y culturales.

Este derecho debe entenderse a partir de la equidad de género y no discriminación, tanto en el acceso al líquido vital como en los procesos de toma de decisiones sobre su gestión. Por tanto, debe propender a la construcción de sujetos activos, empoderados en sus espacios locales y realidades; de ahí que este trabajo de investigación versa sobre la importancia de la participación de las mujeres en la gestión comunitaria del agua como medio para garantizar el derecho humano al agua.

Para estos fines, el trabajo parte del reconocimiento del derecho humano al agua a nivel internacional para luego adentrarnos a la realidad normativa ecuatoriana. Posteriormente, se describe la gestión de recursos hídricos en Ecuador, con especial énfasis en las juntas administradoras de agua potable y saneamiento como formas de participación comunitaria. Finalmente, se recogen experiencias sobre la participación de las mujeres en la gestión comunitaria y el desafío que implica la garantía de una participación real, activa y significativa.

1. ONU, Consejo Económico y Social, Cuestiones sustantivas que se plantean en la aplicación del Pacto Internacional de Derechos Económicos Sociales y Culturales: El derecho al agua (artículo 11 y 12 del PIDESC). Observación general $n .^{\circ} 15$ (2002), 20 de enero de 2003, E/C.12/2002/11, https:/www.acnur. org/fileadmin/Documentos/BDL/2012/8789.pdf. 


\section{EL RECONOCIMIENTO DEL DERECHO HUMANO AL AGUA EN LA ESFERA INTERNACIONAL}

El derecho humano al agua y saneamiento tiene su fundamento en el artículo 25 de la Declaración Universal de Derechos Humanos (DUDH) de 1948 y en los artículos 11 y 12 del Pacto Internacional de Derechos Económicos, Sociales y Culturales (PIDESC), normativa que, de manera conjunta con lo establecido en el Pacto Internacional de Derechos Civiles y Políticos (PIDCP), conforman la conocida Carta Internacional de Derechos Humanos. Dichos instrumentos son la base normativa en la arena internacional del Derecho Humano al Agua y Saneamiento. ${ }^{2}$

A nivel internacional, 2010 fue el año en el que la Asamblea General de Naciones Unidas reconoció oficialmente el derecho humano al agua y al saneamiento. En dicha resolución se exhortó a los Estados y organizaciones internacionales a proporcionar recursos financieros para propiciar el aumento de capacidades y la transferencia de tecnología, en particular en los países en desarrollo. ${ }^{3}$ Este reconocimiento implica la construcción de sujetos activos, capaces de exigir al Estado que cumpla con los mínimos establecidos, trascendiendo de una posición pasiva de políticas desarrolladas sin su participación. ${ }^{4}$

En el sistema interamericano, SIDH, el derecho al agua no se encuentra reconocido de forma expresa; sin embargo, el conjunto de sus instrumentos determina una serie de derechos vinculados con el acceso al agua. De hecho, en su informe Anual 2015, la Comisión Interamericana de Derechos Humanos, CIDH, determinó que existe la obligación de los Estados de garantizar el acceso a agua salubre, en cantidad suficiente, como condición para la materialización de los derechos a la vida, integridad personal, salud y el derecho de propiedad de los pueblos indígenas. ${ }^{5}$

En este sentido, la Carta de la Organización de Estados Americanos establece, entre los principios comunes, la eliminación de la pobreza crítica como medio para promocionar y consolidar la democracia. Mientras que, dentro de los objetivos básicos

2. Ralph P. Hall, Barbara Van Koppen y Emily Van Houweling, "The human right to water: the importance of domestic and productive water rights", Sci. Eng. Ethics 20 (2014): 849-68, https://doi.org/10.1007/ s11948-013-9499-3.

3. ONU, Asamblea General, El derecho humano al agua y el saneamiento, 28 de julio 2010, A/RES/64/292, http://www.un.org/ga/search/view_doc.asp?symbol=A/RES/64/292\&Lang=S.

4. Juan Bautista Justo, El derecho humano al agua y saneamiento frente a los Objetivos de Desarrollo del Milenio (ODM) (Santiago de Chile, 2013), 15, https:/www.ohchr.org/documents/issues/water/contributionssustainability/eclac7.pdf.

5. Comisión Interamericana de Derechos Humanos, "Informe anual 2015: Acceso al agua en las Américas. Una aproximación al derecho humano al agua en el Sistema Interamericano”. 
del desarrollo integral, consta la garantía de una nutrición adecuada, así como la generación de condiciones urbanas que hagan posible una vida sana, productiva y digna. ${ }^{6}$

Si bien la Declaración Americana de los Derechos y Deberes del Hombre no reconoce de forma expresa el derecho al agua, el acceso al agua es condición para garantizar el derecho a la vida, integridad personal, salud, alimentación, vivienda y asistencia médica. ${ }^{7}$ Acorde con este pronunciamiento, la Convención Americana sobre Derechos Humanos consagra el derecho a la vida (art. 4), y con ello el deber de los Estados de generar las condiciones necesarias para una existencia digna, así como el derecho a la integridad personal (art. 5) y el derecho a la salud (art. 11). ${ }^{8}$

De su parte, el Protocolo de San Salvador en su artículo 11.1 establece el derecho a vivir en un medio ambiente sano y a contar con los servicios públicos básicos. ${ }^{9} \mathrm{El}$ agua potable se constituye en un servicio esencial para la supervivencia de las personas, por lo que su provisión se entiende implícito en el artículo mencionado. ${ }^{10}$

$\mathrm{Al}$ respecto, cabe mencionar dos Resoluciones adoptadas por la Asamblea General de la OEA en relación con el acceso al agua como un derecho humano. La primera es la Resolución AG/RES.2349 de 2007 sobre "El agua, la salud, y los derechos humanos", en la que se determina que el agua es condición para garantizar la vida y la salud; de modo que, el acceso a agua potable e higiene básica son necesarios para una vida digna. ${ }^{11}$ Por otro lado, la Resolución AG/RES. 2760 de 2012 sobre "El derecho humano al agua potable y al saneamiento", a través de la cual se invitó a los Estados de conformidad con sus realidades nacionales a continuar trabajando para garantizar el acceso a agua potable y saneamiento. ${ }^{12}$

De hecho, la falta de reconocimiento expreso de este derecho no implica que carezca de tutela jurisprudencial por parte de la Corte Interamericana de Derechos Humanos, Corte IDH, ya que son ejemplo sus sentencias dictadas en los casos: Pacheco Teruel y otros vs. Honduras (2012) y Vélez Loor vs. Panamá (2010), en los que la

6. OEA, Asamblea General, Carta de la Organización de los Estados Americanos, 21 de mayo de 1948, http://www.oas.org/es/sla/ddi/tratados_multilaterales_interamericanos_A-41_carta_OEA.asp.

7. Comisión Interamericana de Derechos Humanos, "Informe anual 2015", 489-90.

8. OEA, Asamblea General, Convención Americana sobre Derechos Humanos, 22 de noviembre de 1969, https://www.oas.org/dil/esp/tratados_b-32_convencion_americana_sobre_derechos_humanos.htm.

9. OEA, Asamblea General, Protocolo Adicional a la Convención Americana de Derechos Humanos en materia de Derechos Económicos, Sociales y Culturales, 17 de noviembre de 1988, http:/www.oas.org/ es/sadye/inclusion-social/protocolo-ssv/docs/protocolo-san-salvador-es.pdf.

10. Comisión Interamericana de Derechos Humanos, "Informe anual 2015".

11. OEA, Asamblea General, El agua, la salud y los derechos humanos, 5 de junio de 2007, AG/RES. 2349 (XXXVII-O/07).

12. OEA, Asamblea General, El derecho humano al agua potable y al saneamiento, 5 de junio de 2012, AG/ RES. 2760 (XLII-O/12). 
Corte IDH prevé que todo privado de libertad debe tener acceso al agua potable para su consumo y al agua para su aseo personal, como condición para garantizar su integridad personal. Mientras que, en los casos: Comunidad Indígena Yakye Axa Vs. Paraguay (2006), Comunidad Indígena Sawhoyamaxa vs. Paraguay (2010) y Comunidad Indígena Xákmok Kásek vs. Paraguay (2010), reconoció la titularidad del derecho al agua a los pueblos indígenas. ${ }^{13}$

\section{REGULACIÓN DEL DERECHO HUMANO AL AGUA EN EL ORDENAMIENTO JURÍDICO ECUATORIANO}

En el caso ecuatoriano, la Constitución Política del Ecuador de 1998 fue la primera norma constitucional en vincular el acceso al agua potable con el ejercicio del derecho a la salud y a una vida digna. ${ }^{14}$ Posteriormente, en 2007 en la Asamblea Constituyente de Montecristi se enfrentaron dos formas de entender el mundo: aquellos que defendían la visión centrada en el mercado, para quienes el agua era un recurso para la producción; y aquellos que consideraban el agua un derecho humano fundamental. ${ }^{15}$

La Constitución de la República del Ecuador de 2008 en su artículo 12 reconoció el agua como: a) un derecho humano fundamental; y b) un componente elemental de la naturaleza, sujeto de derechos. En este sentido, el derecho humano fundamental al agua se contempla dentro del apartado de los derechos del Buen Vivir, vinculado, con el ejercicio del derecho a la salud y a una vida digna. Además, el agua es considerada un elemento vital para la naturaleza, sujeto de derechos a la existencia, mantenimiento y regeneración de sus ciclos vitales. ${ }^{16}$

A nivel normativo, en 2014 se promulgó la Ley Orgánica de Recursos Hídricos, Usos y Aprovechamiento del Agua, LORHUyA. Este cuerpo normativo reconoció el acceso al agua como derecho humano fundamental e irrenunciable que implica disponer de "agua limpia, suficiente, salubre, aceptable, accesible y asequible para el uso personal y doméstico en cantidad, calidad, continuidad y cobertura". ${ }^{17}$

13. Comisión Interamericana de Derechos Humanos, "Informe anual 2015".

14. Ecuador, Constitución Politica de la República del Ecuador, Registro Oficial 1, 11 de agosto de 1998.

15. Alberto Acosta y Esperanza Martínez, comps., Agua. Un derecho humano fundamental (Quito: Abya-Yala, 2010), 18.

16. Ecuador, Constitución de la República del Ecuador, Registro Oficial 449, 20 de octubre de 2008.

17. Ecuador, Ley Orgánica de Recursos Hídricos, Usos y Aprovechamiento del Agua, Registro Oficial 305, Suplemento, 6 de agosto de 2014; Andrés Martínez Moscoso et al., "The vital minimum amount of drinking water required in Ecuador", Resources 7, n. ${ }^{\circ} 1$ (24 febrero 2018): 15, https://doi.org/10.3390/resources7010015. 


\section{LA GESTIÓN DE LOS RECURSOS HÍDRICOS EN ECUADOR}

De manera histórica, la gestión del agua en Ecuador se ha regulado mediante tres diferentes modelos: en primer lugar, el privado que dio lugar a los mercados del agua con fines de lucro; en segundo lugar, el de la gestión pública que ejerce el Estado mediante el gobierno central y los gobiernos autónomos descentralizados en Ecuador, respectivamente; $y$, finalmente, el modelo de la gestión comunitaria, en la cual han sido los usuarios organizados los protagonistas (de preferencia indígenas y campesinos). ${ }^{18}$ A continuación en el gráfico se observa la evolución histórica normativa en el marco de los modelos descritos previamente:

\section{Gráfico 1. Evolución histórica de la gestión del agua en Ecuador}

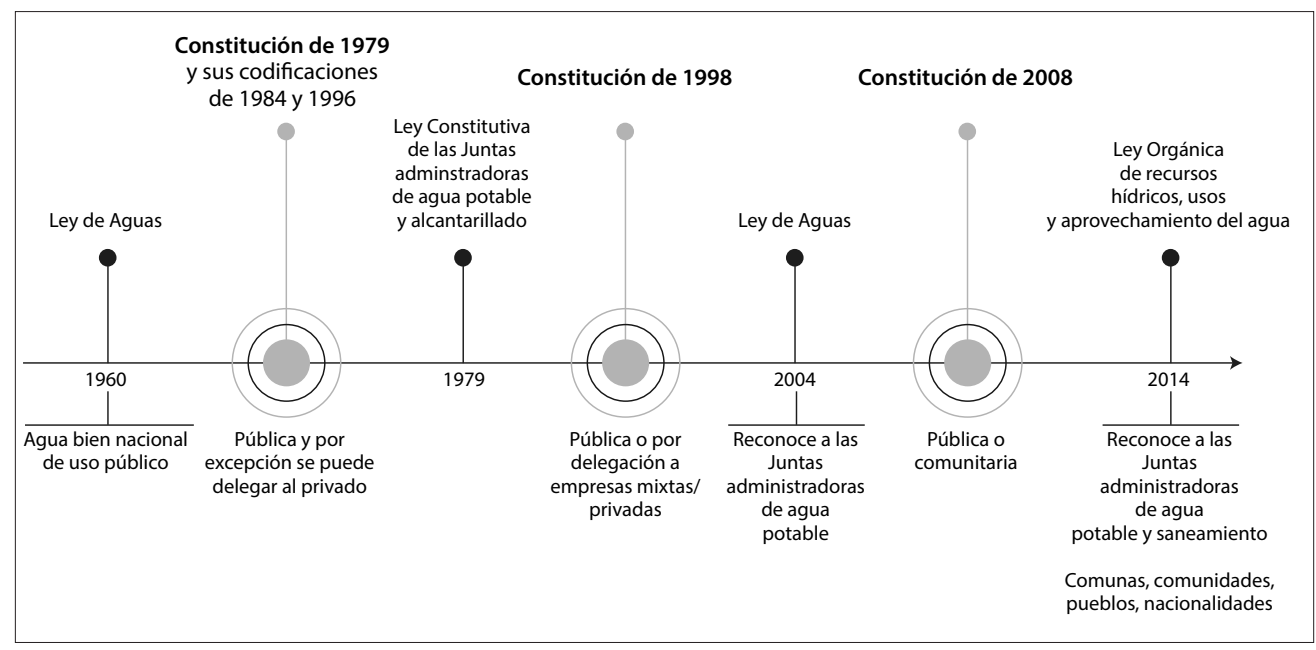

Fuente y elaboración propias a partir de la normativa ecuatoriana.

La norma constitucional de 2008 en su artículo 12 considera el agua como patrimonio nacional estratégico de uso público, y establece la prohibición de privatizar el agua; de modo que el servicio público de saneamiento y el abastecimiento de agua po-

18. Abril Fabiola Casas Cervantes, "La gestión comunitaria del agua y su relación con las políticas públicas municipales. El caso del Manantial de Patamburapio en el Estado de Michoacán, 2009-2014”, Intersticios Sociales, n. ${ }^{\circ} 10$ (2015): 6, http://www.scielo.org.mx/pdf/ins/n10/n10a6.pdf. 
table deben ser provistos únicamente por personas jurídicas estatales o comunitarias. ${ }^{19}$ En concordancia, el artículo 6 de la LORHUyA establece que la gestión es exclusivamente pública o comunitaria, y prohíbe toda forma de apropiación (privatización). Además, determina que la gestión comunitaria les corresponde a las comunas, comunidades, pueblos, nacionalidades, juntas de agua potable y juntas de riego. ${ }^{20}$

Al respecto, la Secretaría Nacional del Agua, Senagua, considera la gestión comunitaria como el conjunto de prácticas, saberes y tradiciones que se mantienen sobre la gestión de los recursos hídricos, su conservación, uso y aprovechamiento. ${ }^{21}$ Mientras que, a nivel latinoamericano, los gestores comunitarios del agua se entienden como estructuras sociales conformadas por vecinos que residen en zonas rurales o periurbanas, en las cuales las empresas públicas, privadas o mixtas no prestan el servicio. Dichas entidades, mediante estatutos de autogobierno, regulan el trabajo común, el pago de tarifas y la elección de dirigentes, además generan sistemas para la captación, la potabilización y la distribución del agua. ${ }^{22}$

\section{LAS JUNTAS ADMINISTRADORAS DE AGUA POTABLE Y SANEAMIENTO, SU NATURALEZA JURÍDICA}

Según la Constitución de 2008, a los gobiernos autónomos descentralizados (GAD) municipales les corresponde prestar los servicios públicos de agua potable y saneamiento ambiental. ${ }^{23}$ Sin embargo, en las localidades rurales en las cuales el GAD municipal no preste el servicio de agua potable, se puede constituir una junta administradora. ${ }^{24}$ Así, la LORHUyA establece que las Juntas Administradoras de Agua Potable y Saneamiento, en adelante Jaaps, son organizaciones comunitarias, sin fines de lucro, que tienen por objeto prestar el servicio público de agua potable. Cabe añadir que dichas organizaciones gozan de autonomía administrativa, financiera y de gestión para cumplir con la prestación efectiva del servicio y el eficaz desarrollo de sus funciones. ${ }^{25}$

19. Ecuador, Constitución de la República del Ecuador, art. 12.

20. Ecuador, Ley Orgánica de Recursos Hídricos, Usos y Aprovechamiento del Agua, art. 6.

21. Senagua, "Gestión comunitaria del agua", 2017, www.agua.gob.ec.

22. Pablo Vagliente et al., Modelos de gobernabilidad democrática para el acceso al agua en América Latina (AVINA, 2011), 23.

23. Ecuador, Constitución de la República del Ecuador, art. 264.

24. Ecuador, Ley Orgánica de Recursos Hídricos, Usos y Aprovechamiento del Agua, art. 32.

25. Ibíd., art. 43 . 
En 2017 Senagua, mediante el Acuerdo n. ${ }^{\circ}$ 31, dictó las directrices y regulaciones para garantizar la permanencia y fortalecimiento de la gestión comunitaria del agua y de la prestación comunitaria de los servicios de agua potable y saneamiento, y riego y drenaje, mediante el cual se ratificó que la gestión comunitaria del agua la cumplen las Jaaps; sin embargo, se establece un régimen de transición para las comunas, comunidades, pueblos y nacionalidades indígenas, pueblo afroecuatoriano y pueblo montuvio al reconocerse sus propias formas de organización y no estar obligadas a constituirse en Jaaps. ${ }^{26}$

Dentro de las funciones de las Jaaps, se encuentran las constantes en el gráfico 2:

\section{Gráfico 2. Deberes y atribuciones legales de las Jaaps}

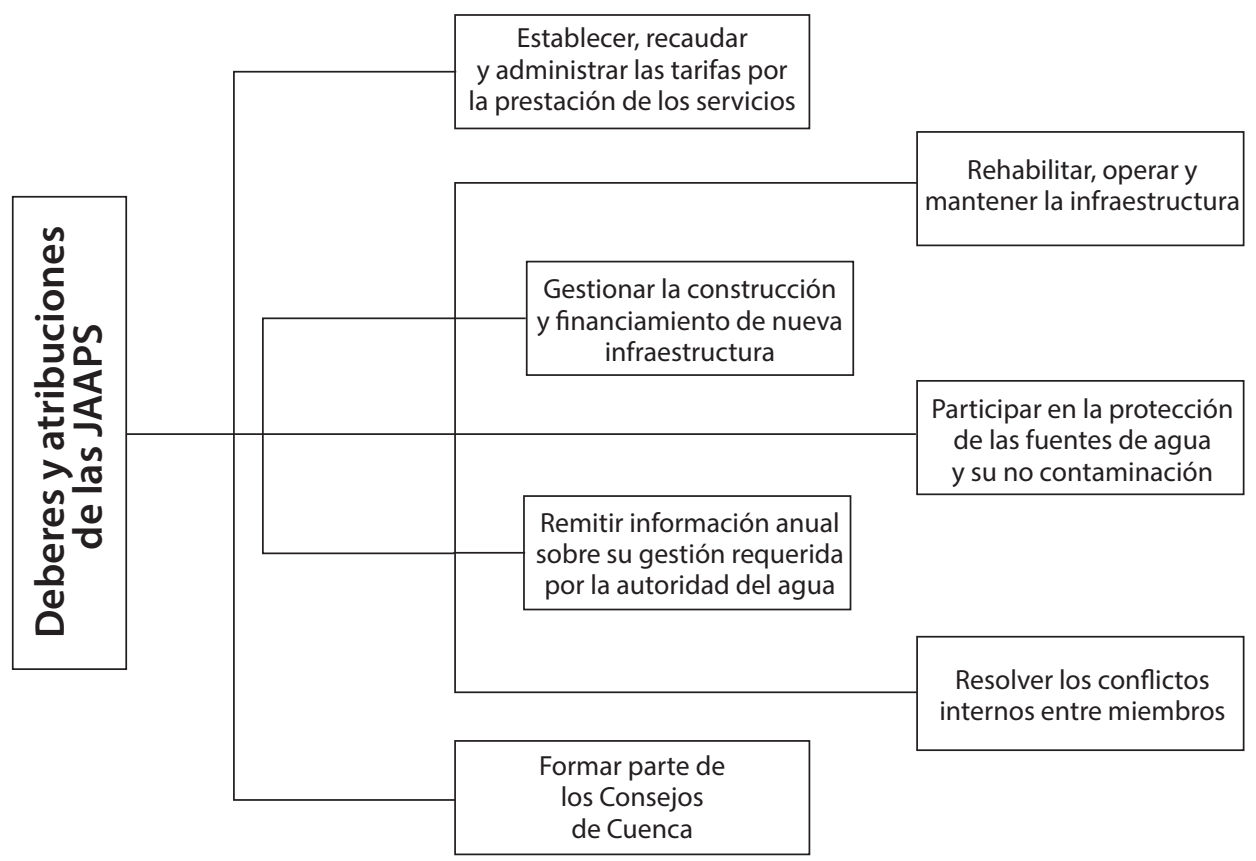

Fuente y elaboración propias a partir del artículo 44 de la LORHUyA (2014).

26. Ecuador, Senagua, Acuerdo Ministerial 31: directrices y regulaciones para garantizar la permanencia y fortalecimiento de la gestión comunitaria del agua y de la prestación comunitaria de los servicios de agua potable y saneamiento; $y$, riego y drenaje, Registro Oficial 88, 27 de septiembre de 2017. 


\section{LA PARTICIPACIÓN COMUNITARIA EN LA GESTIÓN DEL AGUA}

El caso de las Jaaps se encuentra dentro del campo de la participación comunitaria en la cual los actores locales que comparten un territorio y un marco sociocultural se organizan para la provisión del servicio de agua potable, así como para la protección y conservación de las fuentes de abastecimiento. ${ }^{27}$ En este sentido, se puede establecer que el manejo comunitario del agua se desarrolla a través de cuatro principios: la participación y apoyo de todos los sectores de la comunidad; el compartir los costos; el control (indirecto/directo) sobre la operación y mantenimiento de las actividades; $y$, la propiedad colectiva (percibida/real) de la infraestructura. ${ }^{28}$

$\mathrm{Al}$ respecto, cabe señalar que la participación de los actores es clave en la sostenibilidad de los proyectos comunitarios de gestión de agua. ${ }^{29}$ Los enfoques participativos provocan que los diferentes actores se reúnan y expongan sus intereses y posiciones, lo cual conduce a una mejor comprensión de las temáticas y solución de problemas. Así mismo, la participación reviste de mayor legitimidad a las decisiones porque han sido establecidas a partir de un proceso transparente y democrático, provocando que las mismas ocasionen menos resistencia en su aplicación e implementación. ${ }^{30}$

Por otro lado, existen consideraciones negativas en relación con la participación. Una revisión de literatura de Carr, Blöschl, y Loucks nos remite a los trabajos de Karl (2000) y Lubell (2004), en torno a la percepción de que la participación puede consumir tiempo y retardar la toma de decisiones e incluso significar un mayor costo económico para la organización, a pesar de lo cual no se ha podido probar en estudios que exista un vínculo negativo entre la participación y la gestión del agua. ${ }^{31}$ Las críticas surgen dado que, si bien la participación es tan efectiva, por qué las personas en la práctica no lo hacen aun cuando tienen la oportunidad, lo cual nos lleva a la discusión de quiénes participan y bajo qué circunstancias lo hacen.

27. Casas Cervantes, "La gestión comunitaria del agua y su relación con las políticas públicas municipales. El caso del Manantial de Patamburapio en el Estado de Michoacán, 2009-2014", 6.

28. Ryan W. Schweitzer y James R. Mihelcic, "Assessing sustainability of community management of rural water systems in the developing world", Journal of Water, Sanitation and Hygiene for Development 2, n. ${ }^{\circ}$ 1 (March 2012): 21, https://doi.org/10.2166/washdev.2012.056.

29. Ibíd.

30. G. Carr, G. Blöschl y D. P. Loucks, "Evaluating participation in water resource management: a review", Water Resources Research 48, n. ${ }^{\circ} 11$ (2012): 2, https://doi.org/10.1029/2011WR011662.

31. Ibíd. 


\section{LA INCORPORACIÓN DEL ENFOQUE DE GÉNERO EN LA LEGISLACIÓN ECUATORIANA}

La Constitución de 2008 en su artículo 70 contempla la obligación del Estado de implementar política pública con enfoque de género que permita alcanzar la igualdad entre hombres y mujeres. ${ }^{32}$ Bajo este marco, la LORHUyA contempla la relación entre la mujer y el derecho humano al agua, de tal suerte que considera la obligación de incorporar la perspectiva de género en el establecimiento de medidas que atiendan las necesidades específicas de las mujeres en el ejercicio de este derecho.

Además, establece que deberán adoptarse medidas con el objeto de alcanzar la igualdad formal y material entre hombres y mujeres en las actividades de participación comunitaria sobre la gestión del agua, mediante el fortalecimiento de las mujeres como actoras de cambio, ${ }^{33}$ situación que constituye el núcleo central del presente estudio. En este sentido, el 28 de noviembre de 2017 el secretario del Agua dictó el Acuerdo Ministerial n. ${ }^{\circ} 103$, en el cual dispuso la implementación permanente y obligatoria del enfoque de género, interculturalidad y plurinacionalidad en políticas, programas y proyectos sobre la gestión integral de recursos hídricos.

La norma citada determinó que la transversalización de género es una estrategia teórica-metodológica que permite incorporar este enfoque en el marco normativo y de políticas públicas, mediante el análisis de los significados, prácticas, símbolos, representaciones y normas que la sociedad establece a partir de diferencias biológicas, sociales o culturales. De forma expresa indica que se debe promover la participación de las mujeres en las directivas y administración de las Jaaps, para lo que se deben establecer cuotas para asegurar la participación paritaria de las mujeres en la gestión del agua. ${ }^{34}$

En relación con la política pública nacional, la Agenda Nacional de las Mujeres y la Igualdad de Género 2014-2017, elaborada por el Consejo Nacional de Igualdad de Género, consideró en su eje ambiente, propiciar la participación plena de las mujeres y su empoderamiento, de manera especial en la gestión y administración de las juntas de agua potable. ${ }^{35}$ Posteriormente, el mismo instrumento, actualizado a 2018-2021, respectivamente, contempló la urgencia de tomar medidas para alcanzar el empoderamiento de las mujeres rurales para el desarrollo sostenible, a través de la eliminación de barreras que impiden su participación activa en la toma de decisiones y su acceso limitado a los

32. Ecuador, Constitución de la República del Ecuador, art. 70.

33. Ecuador, Ley Orgánica de Recursos Hidricos, Usos y Aprovechamiento del Agua, art. 62.

34. Ecuador, Senagua, Acuerdo 2017-0103, 28 de noviembre de 2017.

35. “Agenda Nacional de las Mujeres y la Igualdad de Género 2014-2017” (Quito, 2014). 
recursos naturales, al agua. Para ello se estableció como política pública incorporar a las mujeres rurales en los espacios e instancias para la vigilancia de la gestión y administración del agua potable y riego, y en la protección de fuentes de agua pura. ${ }^{36}$

\section{EXPERIENCIAS SOBRE LA PARTICIPACIÓN DE LAS MUJERES EN LA GESTIÓN COMUNITARIA DEL AGUA}

Las iniciativas para incorporar la visión de género en la gestión del agua surgen de la consideración de los roles y necesidades diferenciadas entre hombres y mujeres dentro y fuera del hogar. Las diferencias biológicas entre hombres y mujeres determinan necesidades específicas en relación con el agua y el saneamiento. Así, las mujeres tienen acceso especial debido a su salud sexual y reproductiva, de modo que los programas de agua y saneamiento deben considerar la higiene menstrual, la situación de las mujeres embarazadas y de las que acaban de dar a luz, junto con sus bebés. ${ }^{37}$

Además, debido a roles de género impuestos, usualmente las mujeres desempeñan actividades relacionadas con las tareas domésticas, el acarreo del agua para cocinar, servir la comida y bebida, así como para cubrir necesidades higiénicas básicas de la familia, en especial de quienes requieren del cuidado. Por tanto, mejorar las condiciones de acceso al agua y al saneamiento en el hogar tiene consecuencias positivas para el mejoramiento de los contextos en los cuales se ejerce el trabajo doméstico no-remunerado. ${ }^{38}$

De acuerdo a estudios realizados en el sudeste asiático, se evidenció una brecha entre la motivación de las mujeres a actuar y sus habilidades reales para hacerlo. En un análisis de caso en la India, se pudo evidenciar que, mientras el acceso al agua y la compensación financiera motivan la participación de las mujeres, existen factores

36. “Agenda Nacional de las Mujeres y Personas LGBTI 2018-2021" (Quito, 2018).

37. Léo Heller, "Informe del Relator Especial sobre el derecho humano al agua potable y el saneamiento: igualdad de género", 2016, https://documents-dds-ny.un.org/doc/UNDOC/GEN/G16/167/00/PDF/ G1616700.pdf?OpenElement; Catarina de Albuquerque, "Informe de la Relatora Especial sobre el derecho humano al agua potable y el saneamiento, Catarina de Albuquerque: el estigma y el ejercicio de los derechos humanos al agua y el saneamiento", vol. A/HRC/21/4, 2012.

38. Getachew Demie, Mulugeta Bekele y Berhanu Seyoum, "Water accessibility impact on girl and women's participation in education and other development activities: the case of Wuchale and Jidda Woreda, Ethiopia”, Environ Syst Res 5 (2016): 11, https://doi.org/10.1186/s40068-016-0061-6; Caitlin Leahy et al., "Transforming gender relations through water, sanitation, and hygiene programming and monitoring in Vietnam", Gender \& Development 25, n. ${ }^{\circ} 2$ (4 mayo 2017): 283-301, https://doi.org/10.1080/13552074.2 017.1331530 . 
socioeconómicos y socioculturales que limitan la habilidad de las mismas para participar. ${ }^{39}$ En este sentido, en Perú se pudieron observar limitantes vinculadas con la sobrecarga de trabajo en el hogar, escasa capacitación y la no convocatoria de mujeres en la toma de decisiones. ${ }^{40}$

A esto se añade la influencia en la participación de factores como atributos y características personales y del hogar. ${ }^{41}$ Por ejemplo, en la India y Nepal generalizar la categoría "mujeres" resulta preocupante cuando en estas sociedades persiste el sistema de castas que discrimina a las mujeres dalit. ${ }^{42}$

En Bangladesh se desarrollaron proyectos para eliminar la presencia de arsénico en el agua, lo cual buscaba consolidar la participación comunitaria en la gestión de los recursos hídricos. Sin embargo, la participación de las mujeres estuvo marcada por edad, estado civil, educación y clase socioeconómica entre las familias que tenían similar exposición al contaminante. ${ }^{43}$ Por ello, resulta necesario que, en la generación de políticas, se tome en cuenta la composición de género de los grupos, sus relaciones de clase, parentesco, estado civil, con especial atención a las relaciones de poder que se experimente entre hombres y mujeres y cómo estas repercuten de manera diferente en los procesos de inclusión y exclusión. ${ }^{44}$

39. Priyam Das, "Women's Participation in Community-Level Water Governance in Urban India: The Gap Between Motivation and Ability", World Development 64 (1 diciembre 2014): 206-18, https://doi. org/10.1016/J.WORLDDEV.2014.05.025.

40. Water and Sanitation Program, Canadian International Development Agency, and Construcción y Saneamiento Ministerio de Vivienda, "Agua, Género y Ciudadanía", 2007.

41. Das Farhana Sultana, "Rethinking community and participation in water governance", en The Routledge Handbook of Gender and Development, ed. Anne Coles, Leslie Gray y Janet Momsen (Londres: Routledge, 2015), 261-72.

42. Deepa Joshi, "Women, water, caste and gender: the rhetoric of reform in India's drinking water sector", en Globalization of Water Governance in South Asia, ed. Vishal Narain (Nueva Delhi: Taylor \& Francis Group, 2013), 35-52; Stephanie Leder, Floriane Clement y Emma Karki, “Reframing women's empowerment in water wecurity programmes in western Nepal", Gender \& Development 25, n. 2 (4 mayo 2017): 235-51, https://doi.org/10.1080/13552074.2017.1335452.

43. Sultana, "Rethinking community and participation in water governance".

44. Das, "Women's participation in community-level water governance in urban India: The gap between motivation and ability"; Sultana, "Rethinking community and participation in water governance"; Maryann R. Cairns, Cassandra L. Workman e Indrakshi Tandon, "Gender mainstreaming and water development projects: analyzing unexpected enviro-social impacts in Bolivia, India, and Lesotho", Gender, Place \& Culture 24, n. ${ }^{o} 3$ (4 marzo, 2017): 325-42, https://doi.org/10.1080/0966369X.2017.1314945; Leder, Clement y Karki, "Reframing women's empowerment in water security programmes in western Nepal"; J. Boateng y S. Kendie, "Factors influencing the participation of women in rural water supply projects in the asante akim south district", Journal of Arts and Social Science, vol. 3, 2015; B. Agarwal, "Conceptualising environmental collective action: why gender matters", Cambridge Journal of Economics 24, n. 3 (1 mayo 2000): 283-310, https://doi.org/10.1093/cje/24.3.283. 
La experiencia muestra que existe una falsa creencia de que las comunidades son un todo integrado. No obstante, las mismas se caracterizan por presentar en su interior arraigadas jerarquías e inequidades, por lo que, si no se consideran estas realidades, los procesos de participación llegarían incluso a reforzar los patrones de inequidad. En diferentes territorios las mujeres recolectan el agua (muchas deben caminar horas para obtener agua para el uso doméstico), educan a los niños en cuestiones de higiene y entienden las consecuencias de contar con sistemas de saneamiento deficientes. ${ }^{45}$

Por ejemplo, un análisis realizado en Bolivia, Lesoto y la India mostró que las mujeres son en realidad responsables mayoritariamente de las tareas relacionadas con el agua ${ }^{46}$ Del mismo modo, estudios en Tanzania revelaron que la división del trabajo en las zonas rurales ocasiona que las mujeres sean responsables de cocinar, cuidar a los niños y de la higiene doméstica; mientras que, los varones requieren el agua para las actividades productivas. ${ }^{47}$

En Ecuador existe una lógica diferenciada entre hombres y mujeres, fruto de la asignación de roles de género, respecto del uso, administración y control de los recursos naturales; así las mujeres están mayoritariamente relacionadas con los trabajos de cuidado, doméstico no remunerado y la producción de autoconsumo. ${ }^{48}$ La Encuesta Nacional de Uso del Tiempo en 2012 evidenció que en promedio las mujeres ecuatorianas destinaron cuatro veces más tiempo que los varones al trabajo no remunerado, esencialmente en actividades domésticas, ${ }^{49}$ como se ve en el gráfico 3.

Sin embargo, esta brecha es aún más evidente en las zonas rurales, en las cuales las mujeres, respecto de las actividades no remuneradas en el hogar, trabajaban 25:33 semanales más que los varones. ${ }^{50}$

En el gráfico 4, desarrollado a partir de la Encuesta Específica de Uso del Tiempo, ${ }^{51}$ se visualizan los datos desagregados por sexo respecto de la distribución de la participación en actividades productivas de autoconsumo en el área rural.

45. Inter-agency Task Force on Gender and Water (GWTF), Gender, Water and Sanitation: A Policy Brief (Nueva York, 2006), https://bit.ly/2M2L2Yv.

46. Cairns, Workman y Tandon, "Gender mainstreaming and water development projects: analyzing unexpected enviro-social impacts in Bolivia, India, and Lesotho".

47. Christina Geoffrey Mandara, Anke Niehof y Hilje Van Der Horst, "Women and rural water management: token representatives or paving the way to power?", Water Alternatives 10, n. ${ }^{\circ} 1$ (2017): 116-1133, www. water-alternatives.org.

48. "Agenda Nacional de las Mujeres y la Igualdad de Género 2014-2017” (Quito, 2014).

49. INEC, "Encuesta de Uso del Tiempo" (Quito, 2012), http://www.ecuadorencifras.gob.ec//documentos/ web-inec/Uso_Tiempo/Presentacion_Principales_Resultados.pdf.

50. Ibíd.

51. Ibíd. 


\section{Gráfico 3. Número de horas semanales (hh:mm) de trabajo no remunerado desagregado por sexo}

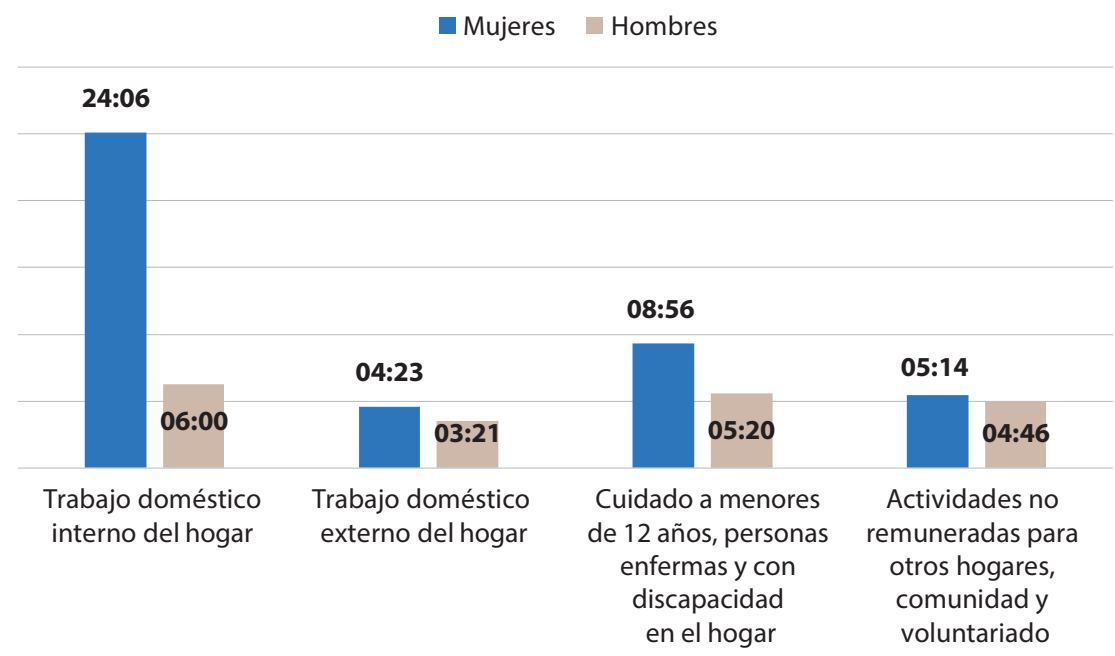

Fuente y elaboración a partir del Consejo Nacional por la Igualdad de Género (2014).

El Banco Interamericano de Desarrollo realizó un estudio sobre prácticas y saberes en la gestión comunitaria del agua en ocho Jaaps en la Costa, Sierra y Oriente del Ecuador. Como conclusión del trabajo, se observó que, si bien las mujeres están más presentes en las actividades colectivas como reuniones, mingas y comisiones, en todos los casos estudiados la presidencia estuvo a cargo de varones, mientras que las mujeres ocupan cargos de secretarias y tesoreras. Adicionalmente, ocurre que en la mayoría de los casos los varones constan como socios usuarios, lo cual condiciona la participación de las mujeres como intermediarias y tramitadoras del servicio. ${ }^{52} \mathrm{~A}$ continuación, consta lo que dijo una mujer ecuatoriana representante de su Jaaps:

Cuando estábamos haciendo campaña para la elección de la junta, nos decían: 'Pero tú, como mujer, ¿qué vas a ir a hacer al pozo?, eso es trabajo de hombres'. Había cierto machismo en nuestras comunidades, pero nosotras, como mujeres, si tenemos que ir a lavar un tubo o hacer limpieza lo hacemos, si tenemos que ponernos botas, lo hacemos. Yo soy

52. María Elena Acosta Maldonado, Marcello Basani y Helder Solíz, Prácticas y saberes en la gestión comunitaria del agua para consumo humano y saneamiento en las zonas rurales de Ecuador (Banco Interamericano de Desarrollo, 2019). 


\section{Gráfico 4. Porcentaje de distribución de la participación de mujeres y hombres en actividades productivas de autoconsumo en el área rural}

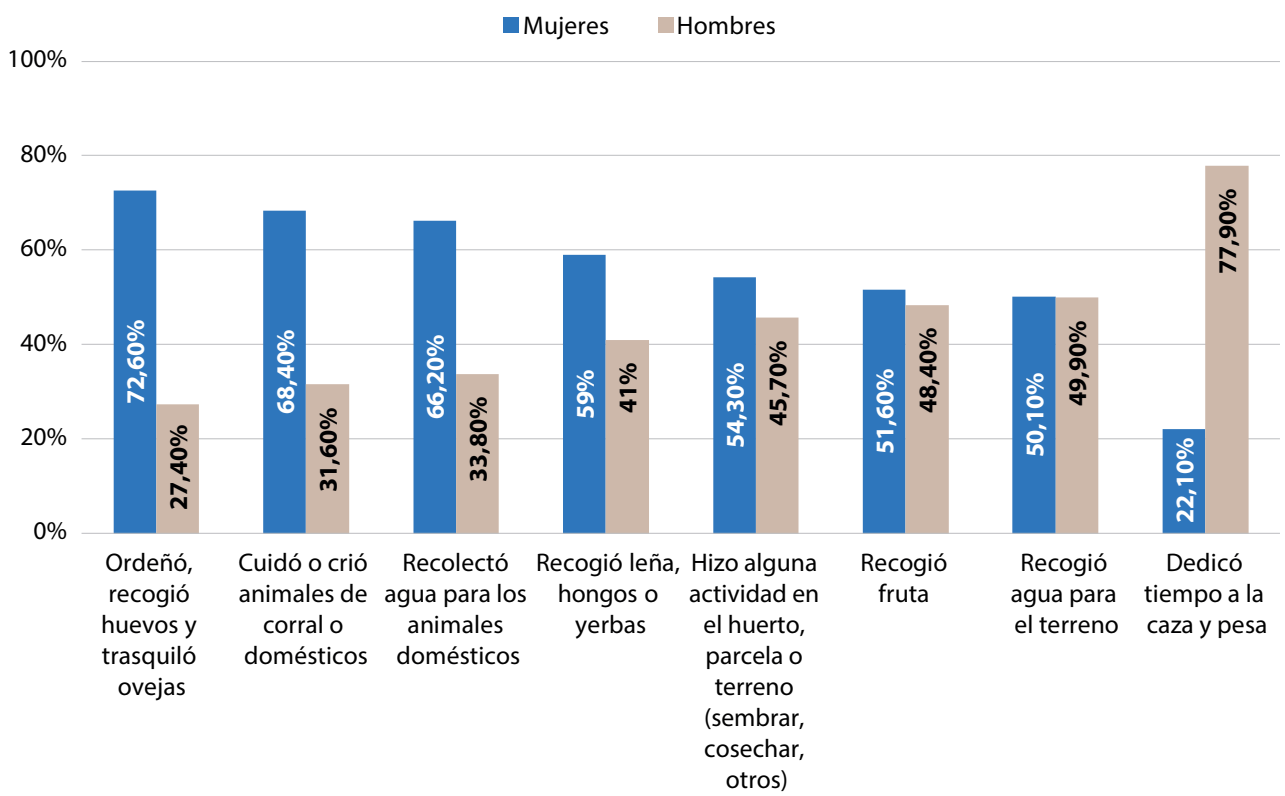

Fuente y elaboración a partir del Consejo Nacional para la Igualdad de Género (2014).

la primera mujer representante de mi comunidad, había mujeres que postulaban, pero no ganaban porque la gente pensaba que debía ser un varón, pero ahora hemos demostrado que se puede (Cecilia Córdova, primera vocal presentante de comunidad Libertador Bolívar, Manglaralto, 2018). ${ }^{53}$

En el área rural, y de manera particular en la gestión de los recursos hídricos, usualmente las mujeres están subrepresentadas en los comités de agua, tanto como usuarias, así como en los puestos clave de toma de decisiones (demostrado a partir de estudios realizados en las zonas rurales de Vanuatu y de Nicaragua). ${ }^{54}$

53. Ibíd.

54. Brecht Mommen, Karen Humphries-Waa y Stanley Gwavuya, "Does women's participation in water committees affect management and water system performance in rural Vanuatu?", Waterlines 36, n. ${ }^{\circ}$ 3 (1 julio 2017): 216-32, https://doi.org/10.3362/1756-3488.16-00026; Clara Murguialday, Avanzando en la equidad de género en la gestión comunitaria del agua. una práctica de planificación conjunta de las ONGD Españolas y la AECID en Nicaragua, 2017, https://www.alianzaporlasolidaridad.org/wpcontent/uploads/Libro-Género-y-Agua-Nica.pdf. 
En Tanzania y Kenia existen referencias sobre sistemas de cuotas implantados en los Comités de Agua para asegurar la participación de las mujeres en los cargos de representación ${ }^{55} \sin$ que esta situación constituya una medida eficiente de una real participación de las mujeres. En este sentido, en Perú se pudo observar limitantes a la participación en la gestión comunitaria, vinculadas con la sobrecarga de trabajo en el hogar, escasa capacitación y la no convocatoria de mujeres en la toma de decisiones.

\section{DESAFÍO REAL PARA LA PARTICIPACIÓN DE LAS MUJERES EN LA GESTIÓN COMUNITARIA DEL AGUA}

La presencia obligatoria de las mujeres no es sinónimo de que sus voces tengan influencia real en los procesos de toma de decisiones, ni que los intereses de estas sean tomados en cuenta. ${ }^{56} \mathrm{Sin}$ embargo, se considera que la inclusión de las mujeres en dichos cuerpos de representación desafía las concepciones socioculturales que asocian los comités con espacios públicos y masculinos, así como la creencia de que el uso del agua doméstica, al ejercerse en los espacios privados, es una cuestión de mujeres. ${ }^{57}$

En general, se sostiene que los roles tradicionales de género se ven expuestos cuando se desarrolla en las mujeres capacidades de gestión del agua, otorgando a estas oportunidades para ocupar roles de liderazgo y mejorar sus condiciones económicas. ${ }^{58}$ Las mujeres que asumen roles en la planificación, diseño e implementación de intervenciones en agua, saneamiento e higiene, atraviesan experiencias de empoderamiento. Por lo tanto, promover programas para la gestión de agua con un enfoque de género influye en el empoderamiento de las mujeres y les da la confianza para que asuman otros roles en la sociedad, además les permite acrecentar su capital social al interior de las comunidades..$^{59}$

55. Niehof Mandara y Van Der Horst, "Women and rural water management: token representatives or paving the way to power?"; Chinwe Ifejika Speranza y Edward Bikketi, "Engaging with gender in water governance and practice in Kenya", en Erratum to: Water Security Across the Gender Divide, ed. Christiane Fröhlich et al. (Londres: Springer International Publishing, 2017), 125-50, https://doi.org/10.1007/978-3319-64046-4_12.

56. Niehof Mandara y Van Der Horst, ibíd.

57. Niehof Mandar y Sultana Van Der Horst, "Rethinking community and participation in water governance".

58. World Water Assessment Programme, Managing water report under uncertainty and risk. The United Nations World Water Development Report 4, UNESCO (París, 2012), https://doi.org/10.1016/0020-0190(95)00060-P.

59. N. Fielmua y D. T. Mwingyine, "Reducing rural poverty through multiple-use water services: the women's perspective in north-western Ghana", Water and Society III 1 (2015): 357, https://doi.org/10.2495/ws150301. 
En general, las mujeres involucradas en dichas intervenciones y el resto de la comunidad observan en esta experiencia la posibilidad de desarrollar destrezas y potencialidades que antes se les había negado. El mejoramiento en su estatus, tanto en sus hogares como en la comunidad, es la oportunidad para que ellas puedan ocupar cargos públicos y roles de influencia, así como, al superarse las dificultades relacionadas con la falta de agua, saneamiento y prácticas de higiene, encontrar nuevas maneras de generar ingresos y ganar independencia financiera. ${ }^{60}$

Adicionalmente, involucrar tanto a hombres como mujeres en el diseño e implementación de políticas relacionadas con el agua tiene efectos respecto de: nuevas y efectivas soluciones en los problemas relacionados con el agua; permite evitar errores costosos e inversiones infructuosas; proyectos más sostenibles; el trabajo en infraestructura de desarrollo tiene un máximo social y económico de retorno; y, contribuye a otras metas de desarrollo como la mortalidad infantil, reducir el hambre y la equidad de género. ${ }^{61}$ Sin embargo, de ello existen críticas en relación a proyectos que, sin considerar las relaciones de poder, fuerzan a las mujeres a ejercer determinados roles en la sociedad, y buscan beneficiarse del conocimiento que han desarrollado las mujeres y centran en ello la razón utilitaria de su participación. ${ }^{62}$

A pesar de que se considera que la participación es positiva para el empoderamiento de las mujeres, la evidencia muestra que muchas de ellas son marginalizadas en el proceso. Así la participación de las mujeres en el proceso de planificación o toma de decisiones sobre la gestión del agua, generalmente percibidos como espacios masculinos, es limitada por las responsabilidades que surgen de la división sexual del trabajo (productivo-reproductivo), el tiempo, el costo, así como por las normas culturales que determinan el comportamiento de las mujeres. ${ }^{63}$

La participación es, entonces, un proceso que se desarrolla en lugares y espacios específicos, que tienen una carga simbólica de género. ${ }^{64}$ La participación de las mujeres se ve usualmente obstaculizada por el horario de las reuniones, las horas de

60. UNICEF; Gender and Water Alliance; Norwegian Ministry of Foreign Affairs, "For her it's the big issue. Putting women at center of water supply, sanitation and hygiene", 2006.

61. Ibíd., 109-10.

62. Cairns, Workman y Tandon, "Gender mainstreaming and water development projects: analyzing unexpected enviro-social impacts in Bsolivia, India, and Lesotho".

63. Sultana, "Rethinking community and participation in water governance"; Verónica Gutiérrez Villalpando et al., "Género y participación de las mujeres en la gestión del agua en las subcuencas río Sabinal y Cañón del Sumidero, Berriozábal, Chiapas", La Ventana. Revista de Estudios de Género, vol. 4 (Universidad de Guadalajara, Centro de Estudios de Género, 2013), http://www.scielo.org.mx/scielo.php?script=sci_arttext\&pid=S1405-94362013000200009.

64. Sultana, ibíd.; Gutiérrez Villalpando et al., ibíd. 
trabajo, las actividades de cuidado o por ser físicamente inaccesible para personas con discapacidades o enfermedades crónicas. ${ }^{65}$

El empoderamiento es un proceso complejo que comprende expandir las habilidades de las personas para tomar decisiones estratégicas en relación con su capacidad de agencia, recursos y logros. De modo que para tomar una decisión es necesario contar con el acceso y control de los recursos. ${ }^{66}$ Por su parte, los logros deben medirse en términos de calidad de vida (salud, vivienda, nutrición). La capacidad de agencia crítica de las personas se mide a través de la habilidad para plantearse una meta (escogida de una diversidad de opciones) y actuar para alcanzarla. ${ }^{67}$

En este marco, cabe indicar que las necesidades estratégicas de género son las necesidades que las mujeres identifican en las relaciones de poder en las cuales se encuentran inmersas, pudiendo tratarse de la división sexual del trabajo, aspectos legales en relación con la violencia doméstica, igual salario y el control de sus cuerpos. ${ }^{68}$ Por otro lado, las necesidades prácticas de género son aquellas que responden a una necesidad percibida de manera inmediata y se relacionan usualmente con el mejoramiento de las condiciones de vida, por ejemplo, en la provisión de agua, salud y empleo. ${ }^{69}$

\section{CONCLUSIONES}

El artículo centra su atención en el análisis normativo con énfasis en los derechos humanos, el derecho humano al agua y la participación de las mujeres en la gestión hídrica a nivel comunitario. Esta particularidad permite visibilizar la interdependencia de los derechos al mostrar la relación íntima entre este derecho, el derecho a la igualdad y no discriminación (por género y por origen étnico o geográfico, de manera específica), que se evidencian en el caso ecuatoriano a partir de la Constitución de Montecristi, debido a la pluralidad jurídica, la interculturalidad y la plurinacionalidad.

La participación que deben tener las mujeres en la gestión de los recursos hídricos comprende un proceso continuo que supera los actos simbólicos de intercambio de información, debiendo procurarse la generación de espacios participativos, con capaci-

65. United Nations Water, "Eliminating discrimination and inequalities in access to water and sanitation", 2015, http://www.unwater.org/publications/eliminating-discrimination-inequalities-access-water-sanitation/.

66. Leder, Clement y Karki, "Reframing women's empowerment in water security programmes in western nepal".

67. Ibíd.

68. Leahy et al., "Transforming gender relations through water, sanitation, and hygiene programming and monitoring in Vietnam".

69. Ibíd. 
tación y acceso a información. Por ello, en las relaciones jerárquicas de poder entre los géneros al interior de las comunidades, los Estados deben garantizar la eliminación de las barreras que impiden que las mujeres rurales participen en igualdad de condiciones en la gestión comunitaria del agua.

Fruto de este trabajo, que centra su análisis en la normativa de la República del Ecuador, se puede colegir que, si bien es cierto que el legislador y el responsable de la política pública en materia hídrica (Senagua) han puesto en valor el rol de la mujer en la gestión del agua, de nada sirve que se legisle y se reconozca esta labor si, en la práctica, a nivel comunitario no existe un cambio de paradigma y una real intención de poner en práctica este cambio de perspectiva.

La real participación comunitaria de las mujeres en la gestión del agua debe verificarse a través de la asistencia a reuniones, mingas, conformación de las directivas, ejercicio del derecho al voto, procesos de rendición de cuentas, en un espacio de pertenencia común que, si bien se ejerce en el espacio público, tiene consecuencias en la satisfacción de una necesidad práctica como es la provisión del servicio de agua potable. No obstante, en estas comunidades subsisten relaciones de poder, fundadas en preconcepciones socioculturales, lo cual se evidencia en la prevalencia de barreras a la participación que impiden que hombres y mujeres participen en igualdad de condiciones.

La participación conlleva un proceso continuo que se verifica a través de la generación de capacidades, acceso a la información y la eliminación no solo de barreras físicas sino también sociales; por ello, es de vital importancia que el Estado ecuatoriano, a través de la Secretaría del Agua, garantice la formación de las mujeres para su empoderamiento a través de la participación comunitaria, pero también se incluya capacitaciones sobre equidad de género, que permita sensibilizar a todos sobre la necesidad de generar espacios inclusivos para la gestión del agua.

Por otro lado, es responsabilidad de la academia, la sociedad civil, y el tercer sector en general poner a disposición de las mujeres la formación que les permita tener un protagonismo en la gestión de los recursos hídricos, así como elaborar evaluaciones independientes de las políticas públicas que buscan la participación comunitaria con perspectiva de género, con el propósito que se cumpla este objetivo y no quede simplemente en letra muerta.

\section{BIBLIOGRAFÍA}

Acosta, Alberto, y Esperanza Martínez, compiladores, Agua. Un derecho humano fundamental. Quito: Abya-Yala, 2010. www.abyayala.org. 
Acosta Maldonado, María Elena, Marcello Basani y Helder Solíz. Prácticas y saberes en la gestión comunitaria del agua para consumo humano y saneamiento en las zonas rurales de Ecuador. Banco Interamericano de Desarrollo, 2019.

Agarwal, B. "Conceptualising environmental collective action: why gender matters". Cambridge Journal of Economics 24, n. 3 (1 mayo 2000): 283-310. https://doi.org/10.1093/ cje/24.3.283.

Boateng, J, y S. Kendie. "Factors influencing the participation of women in rural water supply projects in the Asante Akim South District". Journal of Arts and Social Science. Vol. 3, 2015. https://genderandsecurity.org/sites/default/files/W_and_Rural_Water_Supply_Projects_in_Ghana.pdf.

Cairns, Maryann R., Cassandra L. Workman y Indrakshi Tandon. "Gender mainstreaming and water development projects: analyzing unexpected enviro-social impacts in Bolivia, India, and Lesotho". Gender, Place \& Culture 24, n. 3 (4 marzo 2017): 325-42. https://doi.org/1 0.1080/0966369X.2017.1314945.

Carr, G., G. Blöschl y D. P. Loucks. "Evaluating participation in water resource management: a review". Water Resources Research 48, n. ${ }^{\circ} 11$ (2012): 1-17. https://doi. org/10.1029/2011WR011662.

Casas Cervantes, Abril Fabiola. "La gestión comunitaria del agua y su relación con las políticas públicas municipales. El caso del Manantial de Patamburapio en el Estado de Michoacán, 2009-2014”. Intersticios Sociales, n. ${ }^{\circ} 10$ (2015): 1-43. http://www.scielo.org.mx/pdf/ ins/n10/n10a6.pdf.

Comisión Interamericana de Derechos Humanos. "Informe anual 2015: acceso al agua en las Américas. Una aproximación al derecho humano al agua en el Sistema Interamericano". Washington D. C., 2015. http://www.ianas.org/water/book/diagnostico_del_agua_en_las_ americas.pdf.

Consejo Nacional para la Igualdad de Género. “Agenda Nacional de las Mujeres y la Igualdad de Género 2014-2017”. Quito, 2014.

—. “Agenda Nacional de las Mujeres y Personas LGBTI 2018-2021”. Quito, 2018. https:// www.igualdadgenero.gob.ec/wp-content/uploads/2018/11/Agenda_ANI.pdf-.

Das, Priyam. "Women's participation in community-level water governance in urban India: the gap between motivation and ability". World Development 64 (1 diciembre 2014): 20618. https://doi.org/10.1016/J.WORLDDEV.2014.05.025.

De Albuquerque, Catarina. "Informe de la Relatora Especial sobre el derecho humano al agua potable y el saneamiento: el estigma y el ejercicio de los derechos humanos al agua y el saneamiento". Vol. A/HRC/21/4, 2012.

Demie, Getachew, Mulugeta Bekele y Berhanu Seyoum. "Water accessibility impact on girl and women's participation in education and other development activities: the case of Wuchale and Jidda Woreda, Ethiopia". Environ Syst Res 5 (2016): 11. https://doi.org/10.1186/ s40068-016-0061-6. 
Fielmua, N., y D. T. Mwingyine. "Reducing rural poverty through multiple-use water services: the women's perspective in north-western Ghana". Water and Society III 1 (2015): 353-64. https://doi.org/10.2495/ws150301.

Gutiérrez Villalpando, Verónica, Austreberta Nazar Beutelspacher, Emma Zapata Martelo, Julio Contreras Utrera y Benito Salvatierra Izaba. "Género y participación de las mujeres en la gestión del agua en las subcuencas río Sabinal y Cañón del Sumidero, Berriozábal, Chiapas". La Ventana. Revista de Estudios de Género. Vol. 4. Universidad de Guadalajara, Centro de Estudios de Género, 2013. http://www.scielo.org.mx/scielo.php?script=sci_artte xt\&pid=S1405-94362013000200009.

Hall, Ralph P., Barbara Van Koppen y Emily Van Houweling. "The human right to water: the importance of domestic and productive water rights". Sci. Eng. Ethics 20 (2014): 849-68. https://doi.org/10.1007/s11948-013-9499-3.

Heller, Léo. "Informe del Relator Especial sobre el derecho humano al agua potable y el saneamiento: igualdad de género". 2016. https://documents-dds-ny.un.org/doc/UNDOC/GEN/ G16/167/00/PDF/G1616700.pdf?OpenElement.

Ifejika Speranza, Chinwe, y Edward Bikketi. "Engaging with gender in water governance and practice in Kenya". En Erratum to: water security across the gender divide, editado por Christiane Fröhlich, Giovanna Gioli, Roger Cremades y Henri Myrttinen, 125-50. Londres: Springer International Publishing, 2017. https://doi.org/10.1007/978-3-319-64046-4_12.

INEC. "Encuesta de uso del tiempo". Quito, 2012. http://www.ecuadorencifras.gob.ec//documentos/web-inec/Uso_Tiempo/Presentacion_Principales_Resultados.pdf.

Inter-agency Task Force on Gender and Water (GWTF). Gender, Water and Sanitation: A Policy Brief. Nueva York, 2006. https://bit.ly/2M2L2Yv.

Joshi, Deepa. "Women, water, caste and gender: the rhetoric of reform in India's drinking water sector". En Globalization of water governance in South Asia, editado por Vishal Narain, 35-52. Nueva Delhi: Taylor \& Francis Group, 2013.

Justo, Juan Bautista. El derecho humano al agua y saneamiento frente a los Objetivos de Desarrollo del Milenio (ODM). Santiago de Chile, 2013. https://www.ohchr.org/documents/ issues/water/contributionssustainability/eclac7.pdf.

Leahy, Caitlin, Keren Winterford, Tuyen Nghiem, John Kelleher, Lee Leong y Juliet Willetts. "Transforming gender relations through water, sanitation, and hygiene programming and monitoring in Vietnam". Gender \& Development 25, n. ${ }^{\circ} 2$ (4 mayo 2017): 283-301. https:// doi.org/10.1080/13552074.2017.1331530.

Leder, Stephanie, Floriane Clement y Emma Karki. "Reframing women's empowerment in water security programmes in western Nepal". Gender \& Development 25, n. 2 (4 mayo 2017): 235-51. https://doi.org/10.1080/13552074.2017.1335452.

Mandara, Christina Geoffrey, Anke Niehof y Hilje Van Der Horst. "Women and rural water management: token representatives or paving the way to power?". Water Alternatives 10, n. ${ }^{\circ} 1$ (2017): 116-1133. www.water-alternatives.org. 
Martínez Moscoso, Andrés, Víctor Aguilar Feijó y Teodoro Verdugo Silva. "The vital minimum amount of drinking water required in Ecuador". Resources 7, n. 1 (24 febrero 2018): 15. https://doi.org/10.3390/resources7010015.

Mommen, Brecht, Karen Humphries-Waa y Stanley Gwavuya. "Does women's participation in water committees affect management and water system performance in rural Vanuatu?". Waterlines 36, n. 3 (1 julio 2017): 216-32. https://doi.org/10.3362/1756-3488.16-00026.

Murguialday, Clara. Avanzando en la equidad de género en la gestión comunitaria del agua. Una práctica de planificación conjunta de las ONGD españolas y la AECID en Nicaragua. 2017. https://www.alianzaporlasolidaridad.org/wp-content/uploads/Libro-Género-yAgua-Nica.pdf.

Nunn, Neil. "Puede ser peligroso para el útero: masculinidad hegemónica y el reciclado cooperativo en San Pablo, Brasil'". Gender, Place and Culture 20, n. ${ }^{\circ} 6$ (2013): 794-810. https:// doi.org/10.1080/0966369X.2012.716400.

OEA. Implementación del derecho humano al agua y al saneamiento a través del Programa Interamericano para el Desarrollo Sostenible de la OEA. REDESCA; CIDH; REPÚBLICA PORTUGUESA. 2019. http://www.oas.org/fpdb/press/Derecho_al_agua-printed-version.pdf.

Schweitzer, Ryan W., y James R. Mihelcic. "Assessing sustainability of community management of rural water systems in the developing world". Journal of Water, Sanitation and Hygiene for Development 2, n. ${ }^{\circ} 1$ (marzo 2012): 20-30. https://doi.org/10.2166/washdev.2012.056.

Senagua. "Gestión comunitaria del agua". 2017. www.agua.gob.ec.

Sonia Maria Dias, y Ana Carolina Ogando. "Rethinking gender and waste: exploratory findings from participatory action research in Brazil". Work Organisation, Labour \& Globalisation 9, n. ${ }^{\text {o }} 2$ (2015): 51. https://doi.org/10.13169/workorgalaboglob.9.2.0051.

Sultana, Farhana. "Rethinking community and participation in water governance". En The Routledge Handbook of Gender and Development, editado por Anne Coles, Leslie Gray y Janet Momsen, 261-72. Londres: Routledge, 2015. http://farhanasultana.com/wp-content/ uploads/2015/03/Sultana-Handbook-Chapter-2015-Final.pdf.

Unicef, Gender and Water Alliance, Norwegian Ministry of Foreign Affairs. "For her it's the big issue. Putting women at center of water supply, sanitation and hygiene". 2006. https:// www.wsscc.org/wp-content/uploads/2016/04/For-Her-Its-the-Big-Issue-Putting-Womenat-the-centre-of-Water-Supply-Sanitation-and-Hygiene-WASH-Evidence-Report.pdf.

United Nations Water. "Eliminating discrimination and inequalities in access to water and sanitation”. 2015. http://www.unwater.org/publications/eliminating-discrimination-inequalities-access-water-sanitation/.

Vagliente, Pablo, Emilio Ochoa, Lil Soto, Paula Burt, Catarina de Angola y Luciana Ruiz. Modelos de gobernabilidad democrática para el acceso al agua en América Latina. AVINA, 2011. http://www.ecocostas.org/images/documentos/libros/mod-g-d-agua.pdf.

Water and Sanitation Program, Canadian International Development Agency, and Construcción y Saneamiento Ministerio de Vivienda. “Agua, Género y Ciudadanía”. Lima, 2007. 
World Water Assessment Programme. Managing Water report under uncertainty and risk. The United Nations World Water Development Report 4. París: UNESCO, 2012. https://doi. org/10.1016/0020-0chia190(95)00060-P.

\section{Fuentes JURídicas}

Ecuador. Constitución de la República del Ecuador. Registro Oficial 449, 20 de octubre de 2008.

-. Constitución Política de la República del Ecuador. Registro Oficial 1, 11 de agosto de 1998.

- Ley Orgánica de Recursos Hídricos, Usos y Aprovechamiento del Agua. Registro Oficial 305, Suplemento, 6 de agosto de 2014.

Ecuador Senagua. Acuerdo 2017-0103. 28 de noviembre de 2017.

- Acuerdo Ministerial 31: directrices y regulaciones para garantizar la permanencia y fortalecimiento de la gestión comunitaria del agua y de la prestación comunitaria de los servicios de agua potable y saneamiento; y riego y drenaje. Registro Oficial 88, 27 de septiembre de 2017.

OEA. Asamblea General. Carta de la Organización de los Estados Americanos. 21 de mayo de 1948. http://www.oas.org/es/sla/ddi/tratados_multilaterales_interamericanos_A-41_carta_OEA.asp.

-. Convención Americana sobre Derechos Humanos. 22 de noviembre de 1969. https://www. oas.org/dil/esp/tratados_b-32_convencion_americana_sobre_derechos_humanos.htm.

—.El agua, la salud y los derechos humanos. 5 de junio de 2007. AG/RES. 2349 (XXXVII$\mathrm{O} / 07)$.

—. El derecho humano al agua potable y al saneamiento. 5 de junio de 2012. AG/RES. 2760 (XLII-O/12).

- Protocolo Adicional a la Convención Americana de Derechos Humanos en materia de Derechos Económicos, Sociales y Culturales. 17 de noviembre de 1988. http://www.oas. org/es/sadye/inclusion-social/protocolo-ssv/docs/protocolo-san-salvador-es.pdf.

ONU. Asamblea General. El derecho humano al agua y el saneamiento. 28 de julio de 2010. A/ RES/64/292. http://www.un.org/ga/search/view_doc.asp?symbol=A/RES/64/292\&Lang=S.

-. Consejo Económico y Social. "Cuestiones sustantivas que se plantean en la aplicación del Pacto Internacional de Derechos Económicos Sociales y Culturales: El derecho al agua (artículo 11 y 12 del PIDESC)". Observación general, n. ${ }^{\circ} 15$ (2002). 20 de enero de 2003. E/C.12/2002/11. https:/www.acnur.org/fileadmin/Documentos/BDL/2012/8789.pdf. 